INFLAMMATORY BOWEL DISEASE

\title{
PSC-IBD: a unique form of inflammatory bowel disease associated with primary sclerosing cholangitis
}

\author{
E V Loftus Jr, G C Harewood, C G Loftus, W J Tremaine, W S Harmsen, A R Zinsmeister, D A Jewell, \\ W J Sandborn
}

See end of article for authors' affiliations

Correspondence to:

Correspondence to:
Dr E V Loftus Jr, Division of Gastroenterology and Hepatology, Mayo Clinic, 200 First Street, SW Rochester, MN 5590 , USA; loftus.edward@ mayo.edu

Revised version received 18 June 2004

Accepted for publication 22 June 2004
Background: Inflammatory bowel disease associated with primary sclerosing cholangitis (PSC-IBD) may have a high prevalence of rectal sparing, backwash ileitis, and colorectal neoplasia.

Aims: To describe the clinical features and outcomes of PSC-IBD and compare these to a group of chronic ulcerative colitis (CUC) patients.

Methods: The medical records of all patients with PSC-IBD evaluated at the Mayo Clinic Rochester between 1987 and 1992 were abstracted for information on endoscopic and histological features, colorectal neoplasia, surgery, and other clinical outcomes. Patients referred for colorectal neoplasia and those who did not undergo colonoscopy with biopsies were excluded. A control group of CUC patients matched for sex, duration of IBD at first clinic visit, and calendar year of first clinic visit was identified, and similar information was abstracted.

Results: Seventy one PSC-IBD patients and 142 CUC patients without PSC were identified. Rectal sparing and backwash ileitis were more common in the PSC-IBD group (52\% and $51 \%$, respectively) than in controls ( $6 \%$ and $7 \%$, respectively). Overall, colorectal neoplasia developed in 18 cases and 15 controls, including 11 cancers (seven cases and four controls). An increased risk of colorectal neoplasia or death was not detected in a matched analysis. Although the cumulative incidence of colorectal neoplasia was higher in cases (33\%) than in controls (13\%) at five years, this was of borderline statistical significance ( $p=0.054$, unmatched log rank test). Overall survival from first clinic visit was significantly worse among cases $(79 \% \vee 97 \%)$ at five years ( $p<0.001$, unmatched log rank test).

Conclusion: PSC-IBD is frequently characterised by rectal sparing and backwash ileitis. Colorectal neoplasia develops in a substantial fraction and overall survival is worse. PSC-IBD may represent a distinct IBD phenotype.
$\mathrm{P}$ rimary sclerosing cholangitis (PSC) is a chronic cholestatic disease of unknown aetiology, characterised by inflammation and fibrosis of the biliary tree. ${ }^{1-3}$ PSC may eventually progress to hepatic cirrhosis and subsequent liver failure, and is accompanied by an increased risk of cholangiocarcinoma. ${ }^{45}$ PSC may occur in the presence or absence of inflammatory bowel disease (IBD). The prevalence of IBD (typically chronic ulcerative colitis (CUC)) among PSC patients is approximately $70-80 \%$ while only $2-7.5 \%$ of patients with CUC will develop PSC. ${ }^{6}$

IBD associated with PSC (PSC-IBD) remains poorly defined although at least one preliminary report has suggested a high prevalence of pancolitis, with both rectal sparing and "backwash ileitis". ${ }^{7}$ Higher rates of pouchitis following proctocolectomy with ileal pouch-anal anastomosis (IPAA) have also been identified in this population of patients. $^{89}$ PSC may be an independent risk factor for colorectal dysplasia or cancer in patients with CUC but the magnitude of and mechanism of action for this potential risk factor remains unclear. ${ }^{10-24}$ While several studies have shown an increased prevalence of PSC or abnormal hepatic biochemistries in patients with CUC who develop colorectal neoplasia, ${ }^{10}{ }^{12}$ or an increased risk of neoplasia among CUC patients with PSC compared with those without, ${ }^{13} 1618-202224$ others have not. ${ }^{11} 141721$ Differing sample sizes, end points, and comparison groups may have contributed to these disparate results. ${ }^{25}{ }^{26}$ These distinguishing clinical features between PSC-IBD and CUC suggest that PSC-IBD may be a unique phenotype of IBD.

We addressed these issues by conducting a matched case control cohort study comparing the endoscopic and histological characteristics of IBD in a series of PSC-IBD patients with a matched (two controls per case) group of CUC patients without PSC. We also compared the cumulative incidence of colorectal neoplasia, surgery, and overall survival in these two groups.

\section{METHODS}

The Institutional Review Board of the Mayo Foundation approved the study. A centralised diagnostic index was utilised to identify all patients with PSC and concomitant IBD (cases) evaluated at the Mayo Clinic Rochester between January 1987 and December 1992, and their medical records were reviewed. Conventional colonoscopic and histological criteria were used to diagnose IBD. All cases were required to have had at least one colonoscopy with biopsies at the Mayo Clinic. Conventional radiographic criteria of beading, duct ectasia, and stricturing of the intra- or extrahepatic bile ducts were used to diagnose PSC. ${ }^{27}$ All patients were required to have had a cholangiogram (either retrograde or percutaneous) performed or interpreted at the Mayo Clinic. Liver biopsy findings or elevated hepatic biochemistries alone were not sufficient for a diagnosis of PSC. Cases who had undergone colectomy prior to a diagnosis of PSC or first Mayo visit were excluded from the study. Likewise, cases that had been diagnosed with colorectal dysplasia or cancer elsewhere prior to evaluation at our institution were

Abbreviations: PSC-IBD, inflammatory bowel disease associated with primary sclerosing cholangitis; CUC, chronic ulcerative colitis; OLT, orthotopic liver transplantation; CD, Crohn's disease; IC, indeterminate colitis; PHREG, proportional hazards regression; HR, hazard ratio 
excluded. The control group consisted of CUC patients without PSC evaluated at the Mayo Clinic between 1976 and 1993. Controls were matched to cases for sex, calendar year of first visit to the Mayo Clinic ( \pm 10 years), and duration of IBD prior to first visit ( \pm 5 years).

This study was constructed as a matched case control cohort design where PSC-IBD patients were considered cases and CUC patients without PSC were controls. Both the case and control patients were followed prospectively (through their medical record) for colorectal neoplasia, surgery, and death. This is in contrast, for example, with a retrospective matched case control design where cases were identified as patients with colorectal neoplasia and controls did not have neoplasia.

Medical records were abstracted for the following data: demographic information; duration, extent, and treatment of IBD; duration, extent, and treatment of PSC; colorectal neoplasia (dysplasia and cancer); surgical treatment for IBD; surgical complications such as pouchitis and parastomal varices; orthotopic liver transplantation (OLT); hepatobiliary complications such as cholangiocarcinoma and hepatic failure; duration of follow up; vital status at last follow up; and cause of death. Extent of colitis was defined as the most proximal involvement of inflammatory disease based on histology or endoscopy at any time during the study period. Rectal sparing was rectal mucosa that was either endoscopically or histologically normal, or endoscopically and histologically less inflamed than more proximal mucosa. Backwash ileitis was defined endoscopically as a patulous ileocaecal valve with increased granularity of the terminal ileal mucosa. A normal ileal biopsy excluded the diagnosis of backwash ileitis even if the above endoscopic findings were noted. For patients diagnosed with "Crohn's disease" (CD) or "indeterminate colitis" (IC), endoscopic and histological features of all available colonoscopies or sigmoidoscopies were re-reviewed for evidence of CD or IC.

The association of clinical characteristics which might have an association with PSC case status was assessed by conditional logistic regression models using a generalised estimating equations approach in SAS procedure proportional hazards regression (PHREG) to account for the potential correlations within matched sets of cases and one or more controls (using a simple modification of the SAS procedure $\mathrm{PHREG}^{28}$ ).

For survival, cases were considered to be "at risk", beginning at their analysis start date (the latest of the following three dates: first Mayo Clinic visit, diagnosis of IBD, or diagnosis of PSC). Controls were considered "at risk" beginning at the later of first Mayo Clinic visit or diagnosis of UC. This date is subsequently referred to as the "observational start date."
The primary analysis examined survival free of an event for these four outcomes: (1) colorectal cancer alone; (2) colorectal cancer or dysplasia; (3) subsequent proctocolectomy; and (4) patient death. Each analysis accounted for the matching of PSC-IBD cases to CUC controls using survival models for a matched case control design (SAS procedure PHREG $^{28}$ with case control matched sets as strata). For three end points (colorectal cancer alone, colorectal cancer or dysplasia, and subsequent proctocolectomy), the additional relevant covariates of patient age, duration of IBD, and date of IBD diagnosis were included in the models. (Although the latter two factors were used to match a case to two controls, they were not exactly matched.) For overall survival, as there were only 15 events, a model including both age and case control status failed to converge. Hazard ratios (HR) and 95\% confidence intervals (CI) were computed from the estimated coefficients in the PHREG models. In this analysis of overall survival, follow up was censored at the time of the last follow up or at OLT. (Censoring overall survival data at the time of liver transplantation is appropriate as this procedure significantly alters the "natural history" of chronic liver diseases such as PSC.)

Secondary analyses of event free survival ignored the matching between cases and controls and also used Cox PHREG models ${ }^{29}$ (SAS procedure PHREG $^{28}$ ). However, to assess whether a diagnosis of PSC was significantly associated with each outcome, these analyses also included the potential effect modifiers of age at observational start date, calendar year of start date, duration of IBD at start date, and the presence of rectal sparing as additional covariates (predictor variables) in each event type model. The cumulative probability of colorectal neoplasia, subsequent proctocolectomy, and overall survival, separately for cases and controls, were estimated using the Kaplan-Meier product limit method, ${ }^{30}$ and a univariate comparison of these groups was examined using the two sample log rank test. In the unmatched analysis of overall survival, follow up was censored at the time of the last follow up or at OLT.

\section{RESULTS}

\section{Demographics and extent of colitis}

In total, 71 patients with concurrent PSC and IBD were identified, and these were matched with 142 UC patients without liver involvement. Of 71 cases, 61 (86\%) had CUC, five were diagnosed with CD (7\%), and five patients had IC (7\%). Table 1 displays the features of the three IBD subgroups. As noted in the footnote to table 1, only two of five patients carrying a diagnosis of $\mathrm{CD}$ had clearcut evidence of this (colonic stricture and perianastomotic ulcers in one, and perianal fistula and ileal stricture in the other). The other three " $\mathrm{CD}^{\prime}$ patients had in fact indeterminate features, and

Table 1 Endoscopic characteristics of inflammatory bowel disease among PSC-IBD cases

\begin{tabular}{lllll}
\hline & $\begin{array}{l}\text { PSC-UC } \\
(\mathbf{n}=61)\end{array}$ & $\begin{array}{l}\text { PSC-CD* } \\
(\mathbf{n}=5)\end{array}$ & $\begin{array}{l}\text { PSC-IC† } \\
(\mathbf{n}=5)\end{array}$ & $\begin{array}{l}\text { Total } \\
(\mathbf{n}=71)\end{array}$ \\
\hline Pancolitis & $56(92 \%)$ & $2(40 \%)$ & $4(80 \%)$ & $62(87 \%)$ \\
Rectal sparing & $32(52 \%)$ & $3(60 \%)$ & $2(40 \%)$ & $37(52 \%)$ \\
lleitis & $19 / 37(51 \%)$ & $3 / 4(75 \%)$ & $1 / 4(25 \%)$ & $23 / 45(51 \%)$ \\
\hline
\end{tabular}

*These patients were found to have: (1) colonic stricture requiring right hemicolectomy, later with ulcers at anastomosis; (2) perianal fistula, ileal stricture, and contracted caecum; (3) aphthous ulcers in the distal colon; (4) pancolitis which was patchy on biopsy; and (5) linear ulcers on one colonoscopy but pancolitis on four subsequent procedures.

†These patients were found to have: (1) aphthous ulcers throughout the colon; (2) linear ulcers on one colonoscopy but pancolitis seen subsequently; (3) focal inflammation endoscopically in the descending colon and hepatic flexure but diffuse inflammation on biopsy; (4) proximal colitis elsewhere but pancolitis here; and (5) patchy histological inflammation with a single granuloma, diffuse pancolitis on subsequent examinations.

PSC-IBD, inflammatory bowel disease associated with primary sclerosing cholangitis; CD, Crohn's disease; IC, indeterminate colitis; UC, ulcerative colitis. 
Table 2 Clinical features and outcomes of PSC-IBD (cases) and controls with CUC

\begin{tabular}{lcc} 
& PSC-IBD & Comparison CUC \\
& $(\mathbf{n = 7 1 )}$ & (n=142) \\
\hline Demographics and clinical features & & \\
Males (n (\%)) & $46(65 \%)$ & $92(65 \%)$ \\
Age at IBD diagnosis (y) (median (range)) & $32(9-73)$ & $28(6-80)$ \\
Age at PSC diagnosis (y) (median (range)) & $42(10-71)$ & NA \\
Pancolitis (n (\%)) & $62(87 \%)$ & $76(54 \%)$ \\
Rectal sparing (n (\%)) & $37(52 \%)$ & $8(6 \%)$ \\
Ileitis (n (\%)) & $23 / 45(51 \%)$ & $10(7 \%)$ \\
Outcomes & $18(25 \%)$ & $15(11 \%)$ \\
CRN total (n (\%)) & $8(11 \%)$ & $7(5 \%)$ \\
Low grade dysplasia (n (\%)) & $3(4 \%)$ & $4(3 \%)$ \\
High grade dysplasia (n (\%)) & $7(10 \%)$ & $4(3 \%)$ \\
Colorectal cancer (n (\%)) & $12.7(0.3-41)$ & $12.1(1$ day-5.8) \\
IBD diagnosis to CRN (y) (median (range) interval) & $4.9(0.03-20)$ & NA \\
PSC diagnosis to CRN (y) (median (range) interval) & $31(28 \%)$ & $66(46 \%)$ \\
Surgery (n (\%)) & $14(13 \%)$ & $43(30 \%)$ \\
IPAA (n (\%)) & $5(7 \%)$ & $21(15 \%)$ \\
Brooke ileostomy (n (\%)) & $7(6 \%)$ & $0(0 \%)$ \\
Ileorectal anastomosis (n (\%)) & $10 / 14(71 \%)$ & $13 / 30(30 \%)$ \\
Pouchitis (n (\%)) & $2 / 5(40 \%)$ & $0(0 \%)$ \\
Stomal varices (n (\%)) & & \\
\hline PSC-IBD, inflammatory bowel disease associated with primary sclerosing cholangitis; CUC, chronic ulcerative \\
colitis; CRN, colorectal neoplasia; IPAA, ileal pouch-anal anastomosis; NA, not applicable.
\end{tabular}

pancolitis was noted in two. Two, possibly three, of the five "IC" patients had features leaning strongly towards CUC. In all three groups of PSC-IBD patients, the prevalence of rectal sparing and backwash ileitis was high.

Table 2 illustrates the matched characteristics of cases and controls. Both groups were matched equally for sex, with a resulting 65\% male predominance. Median age at IBD diagnosis was slightly older in the PSC-IBD group (32 years (range 9-73) $v 31$ years (range 6-80) in the control IBD group). Cases and controls were reasonably well matched with respect to calendar year of diagnosis of IBD (mean (SD) case-control difference, 1.5 (3.3) years) and duration of IBD at the observational start date (mean (SD) case-control difference, 0.8 (2.9) years). Median age at PSC diagnosis in the cases was 42 years (range 10-71). In the cases, median number of years from IBD diagnosis to PSC diagnosis was 8.2 years (range 10.9 years (PSC first) to 32.3 years). The prevalence of pancolitis was $87 \%$ in the PSC group and $54 \%$ in the control CUC group $(\mathrm{p}<0.001)$. Rectal sparing was noted in $52 \%$ in the PSC-IBD group versus $6 \%$ of the CUC control group $(p<0.001)$. Importantly, no patient with rectal sparing was being treated with topical enema preparations

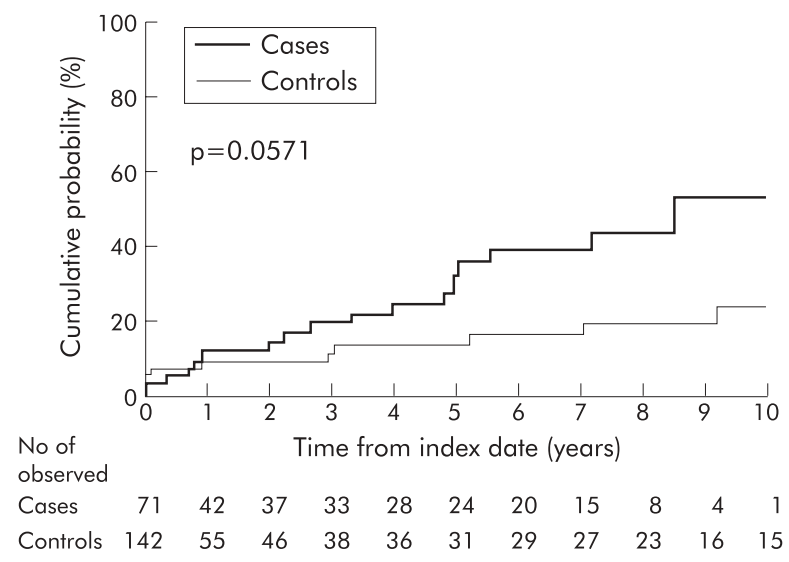

Figure 1 Cumulative probability (1-survival free) of colorectal cancer or dysplasia from the observational start date in cases (those with inflammatory bowel disease associated with primary sclerosing cholangitis) versus controls (those with chronic ulcerative colitis). which might have explained this distribution of colitis. Likewise, "backwash ileitis" was noted significantly more frequently in the PSC-IBD group (51\% of the 45 in whom the ileum was viewed $v 7 \%$ in the control group) $(\mathrm{p}<0.01)$.

\section{Follow up (ignoring matching between cases and controls)}

Subsequent colorectal neoplasia was observed more often in the PSC group (table 2). Seven cases (10\%) and four controls (3\%) developed colorectal cancer, three cases $(4 \%)$ and four controls (3\%) developed high grade dysplasia, and eight cases (11\%) and seven controls (5\%) developed low grade dysplasia. In the cases, two cancers were right sided (39\%) versus two cancers in controls (50\%). In PSC-IBD cases developing cancer or dysplasia, the median interval from diagnosis of IBD to development of dysplasia or cancer was 12.7 years (range 0.3-41). Median interval from first Mayo visit to dysplasia or cancer was three years in PSC-IBD cases (range 8 days -8.5 years). Median time interval from PSC diagnosis to development of dysplasia or cancer was 4.9 years (range 11 days-20 years).

In those cases who did not develop dysplasia or cancer, the median interval between the observational start date (latest of first Mayo visit, IBD diagnosis, or PSC diagnosis) and last follow up or surgical treatment (whichever came first) was 2.0 years (range 1 day-11.4 years). In contrast, the median interval in controls was 0.2 years (range 1 day- 15.5 years) in CUC controls. Considering the time period prior to the first Mayo visit, the median interval from IBD diagnosis to last follow up or surgery was 14.0 years (range 26 days39.7 years) in cases and 10.9 years ( 1 day to 45.4 years) in controls.

IPAA was performed in 14 PSC-IBD patients (13\%) and 43 controls $(30 \%)$. Pouchitis occurred at least once in 10 PSCIBD patients (71\% of those with IPAA) and 13 controls $(30 \%)$. Brooke ileostomy was undertaken in five cases $(7 \%)$ and 21 controls (15\%) while seven PSC-IBD patients $(4 \%)$ underwent subtotal colectomy with ileorectal anastomosis (compared with none of the control patients). Three of five PSC patients who underwent Brooke ileostomy formation developed parastomal varices $(60 \%)$ versus none of the 21 controls who underwent this procedure.

A total of 17 deaths occurred (12 cases, five controls). There were four deaths in the PSC-IBD group from IBD 
related causes (all from metastatic carcinoma) and five deaths from PSC related causes (decompensated liver failure, cholangiocarcinoma, and hepatorenal syndrome). There were three unrelated deaths in the PSC-IBD group, and all five deaths in the control group were unrelated to IBD.

Overall, 23 PSC-IBD cases (32\%) underwent OLT for treatment of PSC. Of these, six $(26 \%)$ developed colorectal neoplasia compared with 12 non-OLT patients $(25 \%)$ who developed colorectal dysplasia or cancer. Bile duct cancer or gall bladder cancer occurred in five patients $(7 \%)$. Two of these patients $(40 \%)$ also developed colorectal neoplasia versus a colorectal neoplasia frequency of $22 \%$ in those cases without hepatobiliary cancer.

\section{Primary analysis retaining matching between cases and controls}

Adjusting for patient age, duration of IBD, and date of IBD diagnosis, a diagnosis of PSC was suggested as a risk factor for colorectal dysplasia or cancer but this was not statistically significant (HR 1.7 (95\% CI 0.6-4.9); $\mathrm{p}=0.29$ ). The association between PSC and development of cancer alone was also not significant (HR 1.9 (95\% CI $0.3-11.9 ; p=0.53)$. A PSC diagnosis was significantly associated with a lower risk for subsequent proctocolectomy (HR 0.3 (95\% CI $0.1-0.7$ ); $\mathrm{p}=0.004$ ). In the univariate analysis, both patient age and a PSC diagnosis were significantly associated with reduced patient survival. However, the limited number of events (only 15 deaths) among the 213 patients precluded including both as variables in the model. Results for overall survival are only given for the unmatched analysis below.

\section{Secondary analysis ignoring matching between cases and controls}

The cumulative probability of colorectal dysplasia or cancer from the observational start date was borderline significantly higher in PSC patients ( $p=0.054, \log$ rank) (fig 1$)$. Five years after the index date, the cumulative probability of dysplasia or cancer was 33\% at five years (95\% CI $17-46 \%$ ) for cases compared with 13\% at five years (95\% CI 4-21\%) for controls. However, the cumulative probability of cancer alone was not significantly different between the two groups (14\% at five years for cases (95\% CI 3-25\%) $v 4 \%$ at five years for controls (95\% CI $0-10 \%) ; p=0.13, \log$ rank).

The PHREG model, incorporating age at the observational start date, calendar year of start date, duration of IBD at start

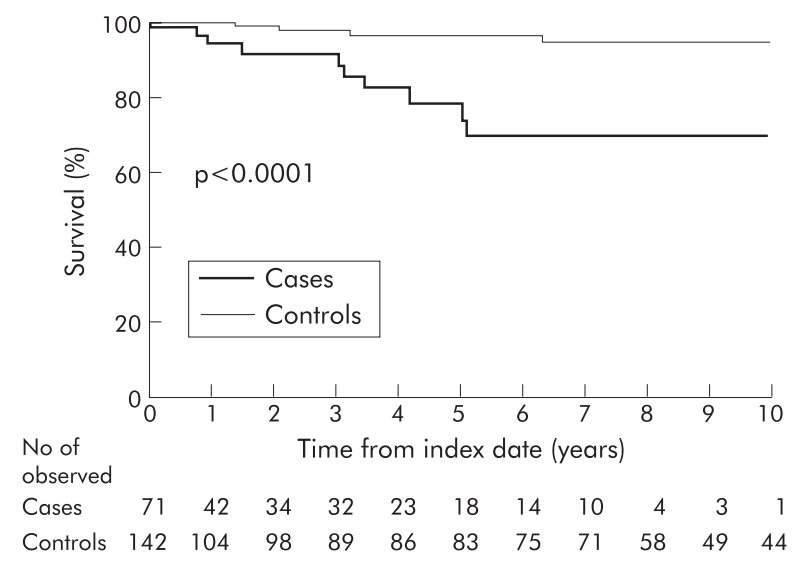

Figure 2 Overall survival from observational start date in cases (those with inflammatory bowel disease associated with primary sclerosing cholangitis) versus controls (those with chronic ulcerative colitis). (Follow up was censored for those who underwent orthotopic liver transplantation.) date, and presence of rectal sparing as potential confounders, indicated that PSC was a borderline significant independent risk factor for the development of dysplasia or cancer (HR 2.1 (95\% CI 0.9-4.7); $p=0.07$ ). Analysis of survival free of cancer alone showed a significantly elevated risk ratio for PSC (HR 4.5 (95\% CI 1.1-18.4); $\mathrm{p}=0.03$ ).

The cumulative probability of proctocolectomy was lower for the PSC group (26\% by five years (95\% CI $13-38 \%$ ) compared with $56 \%$ by five years in the UC group (95\% CI $45-$ $65 \%) ; \mathrm{p}<0.0001, \log$ rank). However, the PHREG model, adjusting for important covariates, indicated that PSC was a borderline significant protective factor (HR 0.5 (95\% CI $0.3-$ $1.0) ; \mathrm{p}=0.054)$.

Overall survival was significantly worse in the PSC-IBD group compared with CUC controls $(p<0.001$, log-rank) (fig 2). Overall survival among cases five years from the observational start date was 79\% (95\% CI 65-94\%) compared with $97 \%$ at five years in controls (95\% CI $93-100 \%$ ). (If follow up was not censored at the time of liver transplantation, the results were not appreciably different-five year survival of $81 \%$ among cases versus $97 \%$ among controls.) The PHREG model adjusting for age at observational start date, calendar year, duration of IBD, and presence of rectal sparing indicated that this association was statistically significant (HR 9.7 (95\% CI 2.3-41); p<0.01).

\section{DISCUSSION}

In this referral centre based study, inflammatory bowel disease associated with primary sclerosing cholangitis (PSCIBD) was characterised by a high prevalence of pancolitis with rectal sparing and backwash ileitis. The study findings were suggestive (but not definite) that PSC-IBD patients were at higher risk for colorectal neoplasia. The primary analysis, based on the matched case control design, failed to indicate a significant increased risk. However, the secondary unmatched analysis adjusting for age, calendar year, and duration of IBD suggested that the presence of PSC was a significant independent risk factor for the development of cancer alone and was suggestive for the development of colorectal neoplasia (cancer or dysplasia). Although PSC-IBD patients were less likely than those of the comparison CUC group to undergo colectomy, the frequency of certain postoperative complications, such as pouchitis or parastomal varices, was higher in these patients. Finally, PSC-IBD patients were also observed to have a reduced survival compared with controls. Again, the matched analysis failed to confirm this while the unmatched analysis indicated that the presence of PSC was a significant independent risk factor for death adjusting for several potential confounders. All of the above distinguishing characteristics suggest that PSC-IBD may be a distinct IBD phenotype.

Most large case series of PSC patients have focused on the hepatobiliary manifestations of the condition, and there are surprisingly few details about the extent of PSC-IBD. ${ }^{1231-33}$ Most PSC-IBD patients have extensive colitis, with or without ileal involvement. The prevalence of isolated ileal involvement is quite low. The high prevalence of rectal sparing in PSC-IBD has been reported in preliminary fashion. ${ }^{7}$ In many series, this subtype of IBD is thought to represent "IC" on the basis of either ileal involvement or rectal sparing. The prevalence of $\mathrm{CD}$ reported in the same series ranges from $0 \%$ to $17 \%$, with an average of $8 \% .{ }^{1231-33}$ Where specified, most of these patients with "CD" have colonic involvement. Most of these patients do not appear to have features that are strongly suggestive of $C D$, such as fistulas, deep ulcers, or granulomas. It is not clear if the variance in prevalence of IBD subtypes is related to a true difference in prevalence or a misclassification of subtype. At our institution, PSC patients with rectal sparing or mild ileitis 
in the setting of pancolitis were not thought to have Crohn's colitis or IC, but rather CUC with rectal sparing, or "PSCIBD."

Our study findings do not resolve the issue of whether PSC could be an independent risk factor for colorectal neoplasia in IBD. In an earlier cohort study from our institution, the number of colorectal cancers in a cohort of PSC patients (which did not overlap with the cohort in the present study) was compared with that expected based on rates of colorectal cancer in a Swedish population based cohort. ${ }^{17}$ The use of population based controls from another country for referral based cases may not have been appropriate. In addition, the cumulative colectomy rate was quite high $(38 \%)$ and this may have masked the propensity for development of neoplasia. ${ }^{17}$ In the present study, we utilised a comparison CUC group selected from the same referral population as the PSC-IBD cases. In a previous case control study from our institution, the prevalence of PSC among CUC cases with colorectal cancer was compared with that among CUC controls without colorectal cancer. ${ }^{21}$ The adjusted odds ratio for colorectal carcinoma with PSC was 1.23 (95\% CI $0.62-$ 2.42). However, the fact that our institution serves as a major referral centre for PSC may have artificially inflated the prevalence of PSC among CUC controls.

The mechanism by which PSC could be associated with an increased risk of colorectal neoplasia in IBD remains unclear. Increased duration and extent of colitis are well recognised risk factors for dysplasia and cancer, and there is a high prevalence of pancolitis in PSC-IBD. After adjusting for duration of IBD, the association of PSC with the development of dysplasia and cancer was only of borderline statistical significance. Disease activity of IBD in PSC patients is often mild and occasionally completely asymptomatic. ${ }^{74}{ }^{35}$ Indeed, in one study, PSC patients without bowel symptoms were found to have not only pancolitis but also indefinite or low grade dysplasia, suggesting that the pancolitis was longstanding. ${ }^{34}$ Therefore, it is possible that PSC serves as a surrogate marker for longstanding but asymptomatic CUC. Other possible mechanisms for the association may include alterations in the bile salt pool or folate deficiency. The intriguing finding of lower rates of colorectal neoplasia among PSC patients treated with ursodeoxycholic acid suggests that bile acids indeed may play a role in the pathogenesis, ${ }^{36}{ }^{37}$ and this warrants further study. What is not in doubt is the 10-fold increased risk of colorectal cancer in PSC patients relative to the general population. ${ }^{38}$ For the clinician, it seems prudent to recognise that PSC-IBD patients clearly have a substantial risk of colorectal dysplasia or cancer, regardless of the mechanism of action, and such patients should be enrolled in a colonoscopic surveillance programme immediately on diagnosis of PSC.

The difference in the rates of colectomy between cases and controls suggests a milder course of colitis in PSC-IBD patients compared with UC patients, which has been noted previously, ${ }^{7335}$ but it is conceivable that referral bias influenced the results. Pouchitis following IPAA appeared to be more common in PSC-IBD cases, confirming previous observations. ${ }^{8}{ }^{9}$ The mechanism for the association between PSC and pouchitis remains unclear. The high rate of parastomal varices in those cases that underwent Brooke ileostomy formation is more easily explained on the basis of portal hypertension. Surgical revision of the stoma alone is usually unsuccessful, and these patients usually require a procedure to address the underlying portal hypertension, such as TIPS, surgical shunting, or liver transplantation. ${ }^{8}$

The increased mortality in the PSC group can be explained mostly by IBD and PSC related causes (colorectal cancer, hepatic failure, and cholangiocarcinoma). Cholangiocarcinoma remains an important cause of morbidity and mortality in PSC; bile duct cancer developed in 7\%, similar to other studies. ${ }^{45}$

Several weaknesses of our study deserve comment. Firstly, our study relied on review of the medical records of patients retrospectively identified from diagnostic indices. Therefore, endoscopic surveillance for neoplasia was not performed on a protocol basis and was variable in frequency. Secondly, because PSC is a disease with an insidious onset and a presumable lengthy asymptomatic phase, the date of diagnosis of PSC is to some extent driven by the awareness and diagnostic aggressiveness of individual physicians. Therefore, patients in whom colorectal cancer was diagnosed several years before or simultaneously with a diagnosis of PSC may actually have had asymptomatic PSC for years. Thirdly, because the subclinical phase of PSC-IBD may be longer than previously appreciated, the duration of colitis in the PSC population may have been similarly underestimated. Fourthly, the high prevalence of pancolitis, short median interval from diagnosis of IBD to development of neoplasia, and the high cumulative incidence of colectomy in CUC controls suggests that our referral centre based controls may not be representative of CUC in the general population. Finally, the high colectomy rate and shorter follow up after the first Mayo visit in CUC controls may explain in part the differences in results in the matched and unmatched analyses-in the primary analysis, follow up of all members of a case-control pair or trio was censored at the earliest loss of follow up of any member.

Given the high frequency of asymptomatic colitis, rectal sparing, and colorectal neoplasia among PSC-IBD patients, flexible sigmoidoscopy or barium enema would appear to be insufficient means of evaluating the colon. All patients with PSC, even those who are asymptomatic, should undergo full colonoscopy with biopsies to detect subclinical IBD and/or neoplasia. Given the high cumulative incidence of colorectal neoplasia, and short mean interval between PSC diagnosis and development of neoplasia, PSC-IBD patients should consider immediate entry into a surveillance colonoscopy programme. By targeting the subset of patients with CUC at highest risk for dysplasia, surveillance with biopsies may provide the highest yield of patients who have potentially curable disease. Data from this study would suggest that patients with PSC-IBD represent such a subset of patients and require thorough colonoscopic surveillance with extensive biopsy sampling.

\section{Authors' affiliations}

E V Loftus Jr, G C Harewood, C G Loftus, W J Tremaine, D A Jewell, W J Sandborn, Division of Gastroenterology and Hepatology, Mayo Clinic and Foundation, Rochester, MN, USA

W S Harmsen, A R Zinsmeister, Division of Biostatistics, Mayo Clinic and Foundation, Rochester, MN, USA

Conflict of interest: None declared.

Presented in part at the 100th Annual Meeting of the American Gastroenterological Association, Orlando, Florida, May 1999 (Gastroenterology 1999;116:A732) and at the 64th Annual Meeting of the American College of Gastroenterology, Phoenix, Arizona, October 1999 (Am J Gastroenterol 1999;94:2658).

\section{REFERENCES}

1 Wiesner RH, LaRusso NF. Clinicopathologic features of the syndrome of primary sclerosing cholangitis. Gastroenterology 1980;79:200-6.

2 Chapman RW, Arborgh BA, Rhodes JM, et al. Primary sclerosing cholangitis: a review of its clinical features, cholangiography, and hepatic histology. Gut 1980;21:870-7.

3 LaRusso NF, Wiesner RH, Ludwig J, et al. Current concepts. Primary sclerosing cholangitis. N Engl J Med 1984;310:899-903.

4 Wee A, Ludwig J, Coffey RJ Jr, et al. Hepatobiliary carcinoma associated with primary sclerosing cholangitis and chronic ulcerative colitis. Hum Pathol 1985; 16:719-26 
5 Rosen CB, Nagorney DM, Wiesner RH, et al. Cholangiocarcinoma complicating primary sclerosing cholangitis. Ann Surg 1991;213:21-5.

6 Loftus EV, Sandborn WJ, Lindor KD, et al. Interactions between chronic liver disease and inflammatory bowel disease. Inflamm Bowel Dis 1997;3:288-302.

7 Perdigoto R, Wiesner RH, LaRusso NF, et al. Inflammatory bowel disease assoicated with primary sclerosing cholangitis: incidence, severity and relationship to liver disease. Gastroenterology 1991;100:A238.

8 Kartheuser AH, Dozois RR, LaRusso NF, et al. Comparison of surgical treatment of ulcerative colitis associated with primary sclerosing cholangitis: ileal pouch-anal anastomosis versus Brooke ileostomy. Mayo Clin Proc 1996;71:748-56.

9 Penna C, Dozois R, Tremaine W, et al. Pouchitis after ileal pouch-anal anastomosis for ulcerative colitis occurs with increased frequency in patients with associated primary sclerosing cholangitis. Gut 1996;38:234-9.

10 Broome U, Lindberg G, Lofberg R. Primary sclerosing cholangitis in ulcerative colitis - a risk factor for the development of dysplasia and DNA aneuploidy? Gastroenterology 1992; 102:1877-80.

11 Choi PM, Nugent FW, Rossi RL. Relationship between colorectal neoplasia and primary sclerosing cholangitis in ulcerative colitis. Gastroenterology 1992;103:1707-9.

12 D'Haens GR, Lashner BA, Hanaver SB. Pericholangitis and sclerosing cholangitis are risk factors for dysplasia and cancer in ulcerative colitis. Am J Gastroenterol 1993;88:1174-8.

13 Broome U, Lofberg R, Veress B, et al. Primary sclerosing cholangitis and ulcerative colitis: evidence for increased neoplastic potential. Hepatology 1995;22:1404-8.

14 Gurbuz AK, Giardiello FM, Bayless TM. Colorectal neoplasia in patients with ulcerative colitis and primary sclerosing cholangitis. Dis Colon Rectum 1995:38:37-41.

15 Bansal P, Sonnenberg A. Risk factors of colorectal cancer in inflammatory bowel disease. Am J Gastroenterol 1996;91:44-8.

16 Brentnall TA, Haggitt RC, Rabinovitch PS, et al. Risk and natural history of colonic neoplasia in patients with primary sclerosing cholangitis and ulcerative colitis. Gastroenterology 1996;110:331-8.

17 Loftus EV Jr, Sandborn WJ, Tremaine WJ, et al. Risk of colorectal neoplasia in patients with primary sclerosing cholangitis. Gastroenterology 1996;110:432-40.

18 Kornfeld D, Ekbom A, Ihre T. Is there an excess risk for colorectal cancer in patients with ulcerative colitis and concomitant primary sclerosing cholangitis? A population based study. Gut 1997;41:522-5.

19 Leidenius MH, Farkkila MA, Karkkainen P, et al. Colorectal dysplasia and carcinoma in patients with ulcerative colitis and primary sclerosing cholangitis. Scand J Gastroenterol 1997;32:706-11.

20 Marchesa P, Lashner BA, Lavery IC, et al. The risk of cancer and dysplasia among ulcerative colitis patients with primary sclerosing cholangitis. Am J Gastroenterol 1997;92:1285-8.
21 Nuako KW, Ahlquist DA, Sandborn WJ, et al. Primary sclerosing cholangitis and colorectal carcinoma in patients with chronic ulcerative colitis: a casecontrol study. Cancer 1998;82:822-6.

22 Shetty K, Rybicki L, Brzezinski A, et al. The risk for cancer or dysplasia in ulcerative colitis patients with primary sclerosing cholangitis. Am J Gastroenterol 1999;94:1643-9.

23 Aitola P, Mattila J, Matikainen M. Liver involvement in patients operated for ulcerative colitis, with special reference to the association of cholangitis with colorectal dysplasia and carcinoma. Intl J Colorectal Dis 2000;15:167-71.

24 Lindberg BU, Broome U, Persson B. Proximal colorectal dysplasia or cancer in ulcerative colitis. The impact of primary sclerosing cholangitis and sulfasalazine: results from a 20 -year surveillance study. Dis Colon Rectum 2001;44:77-85.

25 Ahnen DJ. Controlled clinical trials: the controls are the key. Gastroenterology $1996 ; 110: 628-30$

26 Jayaram H, Satsangi J, Chapman RW. Increased colorectal neoplasia in chronic ulcerative colitis complicated by primary sclerosing cholangitis: fact or fiction? Gut 2001;48:430-4.

27 MacCarty RL, LaRusso NF, Wiesner RH, et al. Primary sclerosing cholangitis: findings on cholangiography and pancreatography. Radiology 1983; 149:39-44

28 SAS Institute. PHREG, 8th edn. Cary, NC: SAS Institute, 1999

29 Cox DR. Regression models and life-tables (with discussion). J Royal Stat Soc 1972;34:187-220.

30 Kaplan EL, Meier P. Non-parametric estimation from incomplete observations. $J$ Am Stat Assoc 1958;53:457-81.

31 Aadland E, Schrumpf E, Fausa $O$, et al. Primary sclerosing cholangitis: a longterm follow-up study. Scand J Gastroenterol 1987;22:655-64.

32 Stockbrugger RW, Olsson R, Jaup B, et al. Forty-six patients with primary sclerosing cholangitis: radiological bile duct changes in relationship to clinical course and concomitant inflammatory bowel disease. Hepatogastroenterology 1988;35:289-94.

33 Fausa O, Schrumpf E, Elgjo K. Relationship of inflammatory bowel disease and primary sclerosing cholangitis. Semin Liver Dis 1991;11:31-9.

34 Broome U, Lofberg R, Lundqvist K, et al. Subclinical time span of inflammatory bowel disease in patients with primary sclerosing cholangitis. Dis Colon Rectum 1995; 38:1301-5.

35 Lundqvist K, Broome U. Differences in colonic disease activity in patients with ulcerative colitis with and without primary sclerosing cholangitis: a case control study. Dis Colon Rectum 1997;40:451-6.

36 Tung BY, Emond MJ, Haggitt RC, et al. Ursodiol use is associated with lower prevalence of colonic neoplasia in patients with ulcerative colitis and primary sclerosing cholangitis. Ann Intern Med 2001;134:89-95.

37 Pardi DS, Loftus EV Jr, Kremers WK, et al. Ursodeoxycholic acid as a chemopreventive agent in patients with ulcerative colitis and primary sclerosing cholangitis. Gastroenterology 2003;124:889-93.

38 Bergquist A, Ekbom A, Olsson R, et al. Hepatic and extrahepatic malignancies in primary sclerosing cholangitis. J Hepatol 2002;36:321-7.

\section{EDITOR'S QUIZ: GI SNAPSHOT}

\section{Answer}

From question on page 77

The diagnosis is strongyloidiasis hyperinfection. Duodenal biopsy (fig lA, B) shows crypt hyperplasia and villous atrophy. Numerous rhabditiform larvae are seen within the crypts. There is mild to moderate infiltrate of polymorphs in the lamina propia.

Strongyloidiasis stercoralis is a cosmopolitan nematode commonly known as human threadworm. Chronic infection is often asymptomatic and may persist for years or even decades due to the ability of the worm for autoinfection. In an immunocompromised host, the organism multiplies, leading to overwhelming parasitic load (hyperinfection). The treatment of choice in the hyperinfection state is thiabendazole $25 \mathrm{mg} / \mathrm{kg}$ twice a day for 7-10 days. ${ }^{1}$

Strongyloidiasis stercoralis should be considered if at risk patients are commenced on immunosuppressive treatment and fail to improve or deteriorate. The role of duodenal biopsy in the diagnosis of this disease is not well established but it proved to be of great value in our case.

doi: 10.1136/gut.2004.043240

\section{REFERENCE}

1 Mahmoud AAF. Strongyloidiasis. Clin Infect Dis 1996;23:949-53. 